\title{
Magnetic phases of graphene nanoribbons under potential fluctuations
}

\author{
H. U. Özdemir, A. Altıntaş, and A. D. Güçlü \\ Department of Physics, Izmir Institute of Technology, IZTECH, TR35430, Izmir, Turkey \\ (Received 17 November 2015; revised manuscript received 14 December 2015; published 12 January 2016)

\begin{abstract}
We investigate the effects of long-range potential fluctuations and electron-electron interactions on the electronic and magnetic properties of graphene nanoribbons with zigzag edges using an extended mean-field Hubbard model. We show that electron-electron interactions make the edge states robust against potential fluctuations. When the disorder is strong enough, the presence of electron-hole puddles induces a magnetic phase transition from antiferromagnetically coupled edge states to ferromagnetic coupling, in agreement with recent experimental results.
\end{abstract}

DOI: 10.1103/PhysRevB.93.014415

\section{INTRODUCTION}

Graphene [1,2], a two-dimensional honeycomb lattice of carbon atoms, has been the subject of intense investigation for nanoelectronic and spintronic applications due to its high electric and thermal conductivity and intrinsic magnetism [3-8]. Although pure graphene is not expected to be magnetic, if the sublattice symmetry of the honeycomb lattice is broken, there is a possibility to induce magnetism [9]. In particular, atomic-scale engineered graphene nanoribbons with zigzag orientation are expected to exhibit magnetized edges with antiferromagnetic coupling between the opposite edges as confirmed by a large number of theoretical literature [10-21] in agreement with Lieb's theorem [9]. However, most likely due to limited control over edge structure in real applications, direct experimental observation is still lacking. Recently, a semiconductor-to-metal transition as a function of ribbon width was observed in nanotailored graphene ribbons with zigzag edges [22]. This transition is attributed to a magnetic phase transition from the antiferromagnetic configuration to the ferromagnetic configuration, raising hopes for the fabrication of room-temperature graphene-based spintronic devices.

The observation of a magnetic phase transition in graphene nanoribbons is a surprising result due to the experimental difficulties for fabricating clean nanostructures with properly passivated and well defined edges [23-27], and free from imperfections in the lattice or in the substrate. A possible source of irregularity in a graphene structure is the formation of the so-called electron-hole puddles [5,28-30]. Those highly inhomogeneous charge distributions were observed by Martin et al. [31] by mapping the charge neutrality point. Later, Crommie et al. [32] reported that impurities between the substrate and the graphene sheet induce a distorted electron liquid, which is in agreement with earlier theoretical works as well $[28,33]$. A different study stated that corrugations are the mechanism behind the formation of charge inhomogeneities [34]. On the other hand, it was predicted from tight-binding calculations that the presence of electron puddles can mask Anderson localization effects favoring metallic behavior [30].

In this work, we investigate the effect of electron-hole puddles resulting from a long-range potential fluctuation on the edge magnetism of finite nanoribbons, using extended meanfield Hubbard calculations. We show that, electron-electron interactions increase the robustness of edge states against disorder as compared to the tight-binding approach in finite graphene nanoribbons. More importantly, a transition from antiferromagnetic to ferromagnetic edge phase is observed as the strength of the disorder is increased. These results are consistent with a recent experimental observation of a semiconductor-to-metal transition as a function of nanoribbon width [22].

This paper has the following structure. In Sec. II, we introduce the Hamiltonian model describing the nanoribbon system under investigation, electron-electron interactions, and the long-range potential fluctuations. In Sec. III, the results including the effects of disorder potential on the electronic properties within the tight-binding and mean-field models are discussed. The antiferromagnetic-ferromagnetic phase transition is investigated in detail for different disorder configurations and interaction strength. Section IV contains a brief summary.

\section{METHOD AND MODEL}

Our starting point is a single-band tight-binding model for $p_{z}$ orbitals, where $s, p_{x}$, and $p_{y}$ orbitals are neglected as they mainly contribute to the mechanical properties of graphene. Within the mean-field extended Hubbard model, the Hamiltonian is constructed as follows:

$$
\begin{aligned}
H_{\mathrm{MFH}}= & \sum_{i, j, \sigma}\left(t_{i j} c_{i, \sigma}^{\dagger} c_{j, \sigma}+\text { H.c. }\right) \\
& +U \sum_{i}\left(\left\langle n_{i, \uparrow}\right\rangle-\frac{1}{2}\right) n_{i \downarrow}+\left(\left\langle n_{i, \downarrow}\right\rangle-\frac{1}{2}\right) n_{i \uparrow} \\
& +\sum_{i, j} V_{i j}\left(\left\langle n_{j}-1\right\rangle n_{i \downarrow}+\left\langle n_{j}-1\right\rangle n_{i \uparrow}\right) \\
& +\sum_{i \sigma} V_{\mathrm{imp}}(i) c_{i \sigma}^{\dagger} c_{i \sigma} .
\end{aligned}
$$

The first term corresponds to the tight-binding approximation where the hopping parameters $t_{i j}$ are taken to be $t_{\mathrm{nn}}=-2.8 \mathrm{eV}$ for the nearest neighbors and $t_{\mathrm{nnn}}=-0.1 \mathrm{eV}$ for the next-nearest neighbors [1]. The operators $c_{i, \sigma}^{\dagger}$ and $c_{i, \sigma}$ create and annihilate an electron at the $i$ th orbital with spin $\sigma$, respectively. The terms $\left\langle n_{i, \sigma}\right\rangle$ denote the expectation value of electron densities. The second and third terms are the on-site and long-range Coulomb interaction terms, respectively. Here, 

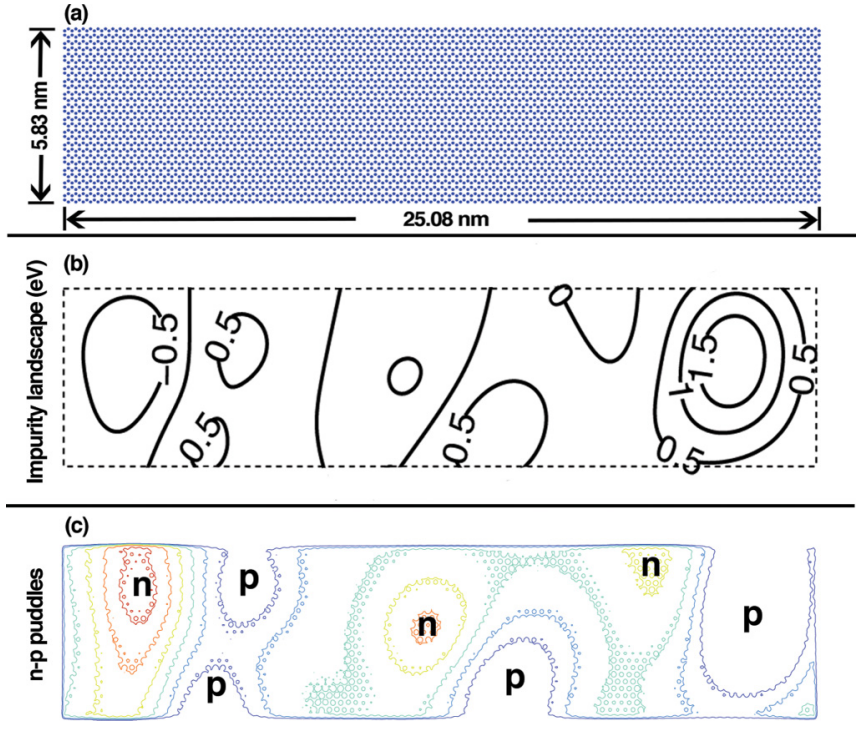

FIG. 1. (a) Graphene nanoribbon lattice structure. (b) Randomly generated impurity potential landscape. (c) Total electron density showing the formation of electron-hole puddles (regions denoted by $n$ and $p$ ), obtained from mean-field Hubbard calculations.

$U$ is taken to be $16.522 / \kappa \mathrm{eV}$, where $\kappa$ is an effective dielectric constant taken to be as a control parameter. The long-range interaction parameters $V_{i j}$ are taken to be $8.64 / \kappa \mathrm{eV}$ and $5.33 / \kappa \mathrm{eV}$ (the Coulomb matrix elements are calculated numerically by using Slater $\pi_{z}$ orbitals [35]) for the first two neighbors, and $1 / d_{i j} \kappa$ for distant neighbors. $V_{\text {imp }}(i)$ represents a smooth long-ranged potential fluctuation, which can be attributed to charge impurities in the substrate.

Our finite structure contains 5740 atoms, respectively, giving rise to about 60 edge states. The length of the lattice vectors are $\left|\vec{a}_{1,2}\right|=0.151 \mathrm{~nm}$. The total length of the ribbon is $25.08 \mathrm{~nm}$ and the width is $5.83 \mathrm{~nm}$ as shown in Fig. 1(a). The mean-field Hamiltonian is solved selfconsistently in the subspaces of the $z$ component of the total spin $S_{z}=\left(n_{\uparrow}-n_{\downarrow}\right) / 2$ (by fixing the number of up and down electrons), and the calculations are performed for several different $S_{z}$ values (see for instance Fig. 5) in order to find the ground-state magnetic configuration. Each calculation was repeated several times starting from different initial density matrices to ensure the convergence to a global energy minimum.

Modeling of the long-range electron-hole puddle disorder, which can be attributed to charged impurities on the structure, is carried out with a superposition of Gaussian electrostatic potentials $V_{\text {imp }}$, which are randomly distributed over the sample, creating a smooth potential landscape [see Fig. 1(b)]. The impurity potential is given by

$$
V_{\mathrm{imp}}(i)=\sum_{n} V_{n} e^{-\frac{\left(\overrightarrow{\vec{r}}_{i}-\overrightarrow{\vec{r}}_{n}\right)^{2}}{2 \sigma^{2}}} .
$$

$V_{n}$ is the potential peak value (randomly chosen between a minimum and a maximum value) of the $n$th impurity located at $\vec{r}_{n}, \sigma$ is the width of the potential, which is taken to be ten times the lattice constant for this study [32]. For such longranged scatterers, Anderson localization effects are expected
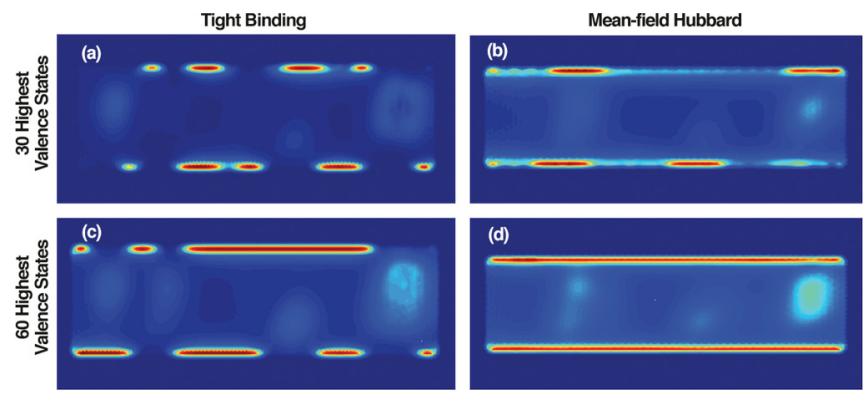

FIG. 2. Electronic density profile corresponding to the 30 highest occupied valence states (top panels), and the 60 highest occupied valence states (bottom panels), obtained using tight-binding (left panels) and mean-field Hubbard calculations (right panels). Electronelectron interactions restore the edge states.

to be suppressed due to the formation of electron-hole puddles [30]. For all calculations, a total of 16 impurity sources are used, and the calculations are repeated for randomly generated configurations. Figure 1(c) shows the formation of electronhole puddles (i.e., negatively and positively charged regions) in the system calculated by subtracting the positive background charge from the total mean-field electron density.

\section{RESULTS}

Before discussing the magnetic properties of the nanoribbons, we first focus on the combined effect of long-range potential fluctuations and electron-electron interactions on the electronic properties of edge states. Figure 2 shows the electronic density profile corresponding to the 30 highest occupied valence states (top panels), and the 60 highest occupied valence states (bottom panels), obtained using tightbinding (left panels) and mean-field Hubbard calculations (right panels), for the disorder configuration given in Fig. 1. We note that in the absence of disorder, valence states include about 30 edge states. In the absence of electron-electron interactions, the main effect of including disorder is to disrupt the edge states, creating highly localized edge states. Note that the (hole) edge states observed in the tight-binding results are not localized in the $\mathrm{p}$ regions indicated in Fig. 1(c). Within the extended Hubbard model, however, an electrostatically more correct spin-dependent filling order of the edge states is obtained, and the hole edge states close to the Fermi level are now located mostly at the $\mathrm{p}$ regions. Another important effect of electron-electron interactions is that the edges states are recovered within the 60 highest valence states. Thus electronelectron interactions make the edge states more robust against disorder by partially restoring the symmetry of the system. The appearance of bulk impurity states is also visible in Fig. 2. An interesting question that arises is how the magnetic properties are affected by the combined effect of disorder and electronic interactions, which will be the focus of the rest of this work.

In Fig. 3, we show the mean-field energy spectra for antiferromagnetic (AFM, top panels) and ferromagnetic (FM, bottom panels) phases, for various degrees of disorder strengths. When no disorder is present, the ground state is AFM and the energy spectrum reveals a gap of the order of $0.17 \mathrm{eV}$, in agreement with previous theoretical work [10,22,36,37] and 

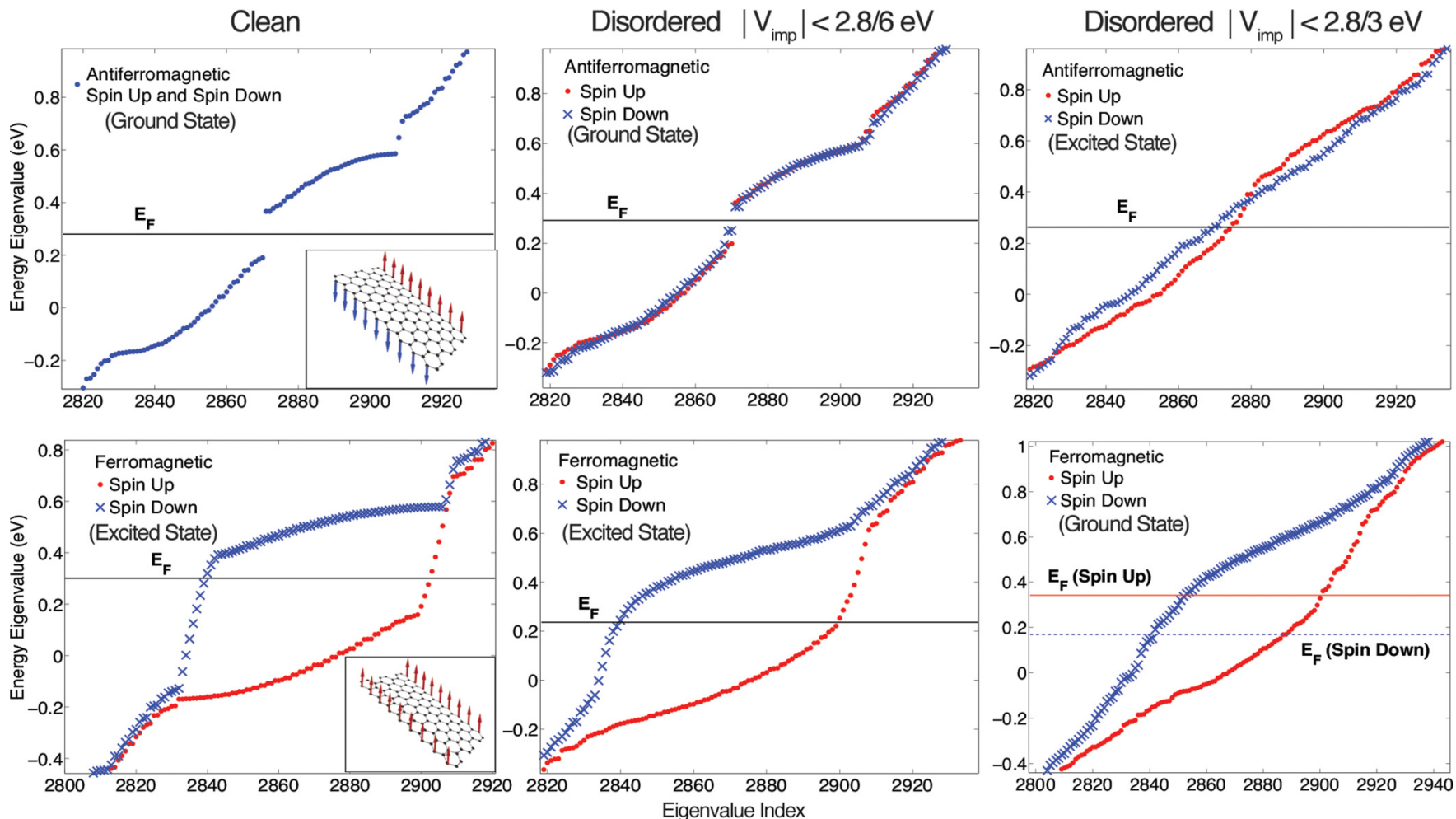

FIG. 3. Mean-field Hubbard spectra for antiferromagnetic (top panels) and ferromagnetic (bottom panels) phases, for various degrees of disorder strengths, characterized by $V_{\text {imp. }} E_{F}$ spin up and spin down show the spin-dependent Fermi levels.

recent experimental results [22]. When disorder is included such that $\left|V_{\mathrm{imp}}\right|<\left|t_{\mathrm{nn}}\right| / 6$, the AFM gap is reduced to $0.1 \mathrm{eV}$, and the ground state is still AFM. However, when the disorder strength is doubled, the AFM gap is practically closed and the system becomes FM. We note that these results are consistent with the experimental results of Ref. [22], where a closing of the gap was observed for a ribbon with widths larger than $7 \mathrm{~nm}$, which was attributed to temperature and doping effects. Here, we show that, although our system is globally charge neutral, local formation of electron-hole puddles due to long-ranged potential fluctuations can also induce an AFM-FM transition.

The results of Fig. 2 were obtained for the particular disorder configuration shown in Fig. 1. In order to check the consistency of the results, we have repeated the calculations for a total of 30 different impurity configurations and strengths. Figure 4(a) shows the energy difference per atom between the AFM and FM phases, a negative value indicating that the ground state is AFM. For impurity strengths $\left|V_{\mathrm{imp}}\right|<\left|t_{\mathrm{nn}}\right| / 6$, no significant effect of disorder is observed. However, for $\left|V_{\mathrm{imp}}\right|<\left|t_{\mathrm{nn}}\right| / 3$, FM phase becomes more dominant. Finally, for $\left|V_{\text {imp }}\right|<\left|t_{\mathrm{nn}}\right|$, all but one out of ten random impurity configurations give a FM ground state. In Fig. 4(b), we plot AFM spectra energy gaps corresponding to the same configurations in Fig. 4(a), showing that the gap quickly decreases as the disorder strength increases.

As discussed earlier, the AFM phase corresponds to $S_{z}=0$ and the FM phase corresponds to $S_{z}=32$. In order to make sure that no other magnetic phases (which could be due to the presence of electron-hole puddles) were not missed in our calculations, we have also performed mean-field calculations for other values of $S_{z}$ between 0 and 35. Figure 5 shows

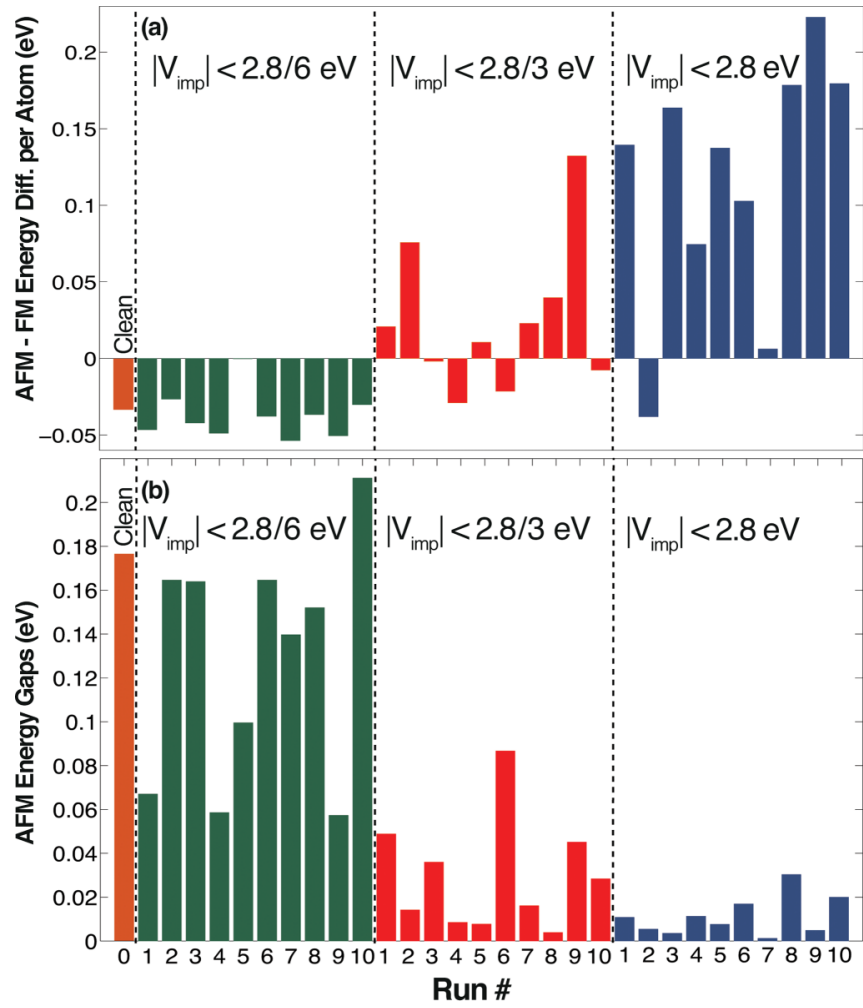

FIG. 4. (a) Energy difference per atom between the AFM and FM phases and (b) the antiferromagnetic phase energy gap for 30 different disorder configurations with various degrees of disorder strengths. A strong disorder effect causes the system to become ferromagnetic. For lower potentials, the chance of a phase transition reduces. 


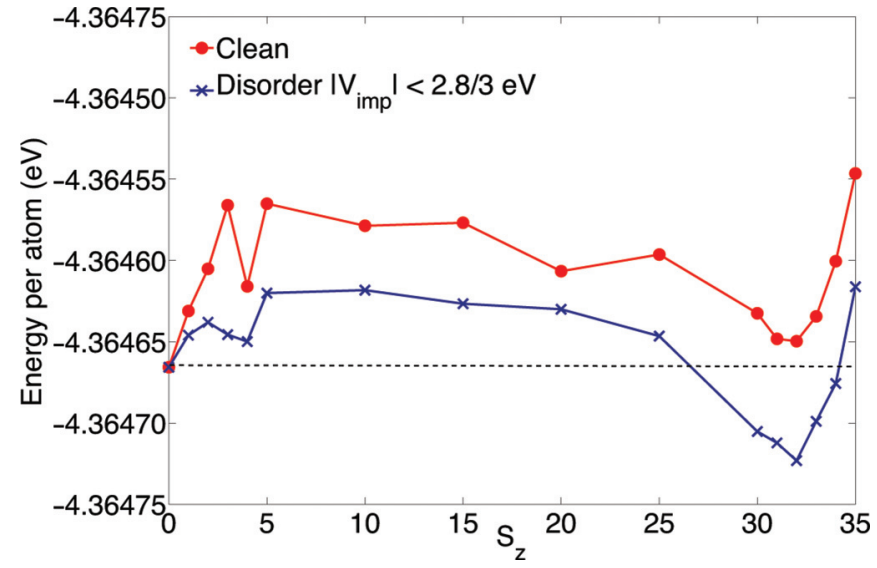

FIG. 5. Total energy of a nanoribbon as a function of magnetization $S_{z}$. For the clean case, the ground state has $S_{z}=0$, and for the disordered case $S_{z}=32$, indicating a FM-AFM phase transition without involving other possible magnetic phases.

the total energy of the clean and disordered nanoribbon as a function of $S_{z}$, for the disorder configuration shown in Fig. 1(b). Clearly, within the mean-field approximation, the most important magnetic states that dominate the low-energy physics are the AFM and FM phases. We observed similar behavior for other disorder configurations as well.

Up to this point, we performed all calculations with $\kappa=6$ whose value determines the magnitude of electronelectron interaction. As there are three main energy variables in our Hamiltonian-hopping parameter, impurity strength, and interaction strength - it is also worth investigating the effect of changing $\kappa$. To see the interplay between $\kappa$ and magnetism, same calculations are performed within the $1 / \kappa=$ $[0.3,0.002]$ interval. A convenient way of investigating the AFM phase is to use staggered magnetism, which is defined as $(-1)^{x}\left(n_{i \uparrow}-n_{i \downarrow}\right) / 2$ where $x$ is even for $\mathrm{A}$ and odd for $\mathrm{B}$ sublattice sites. In Fig. 6, the change of staggered magnetism as a function of dielectric constant is shown. For the clean system, no phase transition is observed in this range. On the other hand, the disordered system shows FM behavior between $1 / \kappa=[0.167,0.04]$. The recovered AFM phase for $1 / \kappa>0.167$ is due to strong electron-electron interactions that suppress the effect of impurities. These results are consistent with our previous results. For the $1 / \kappa<0.04$ region, magnetic properties can be neglected.

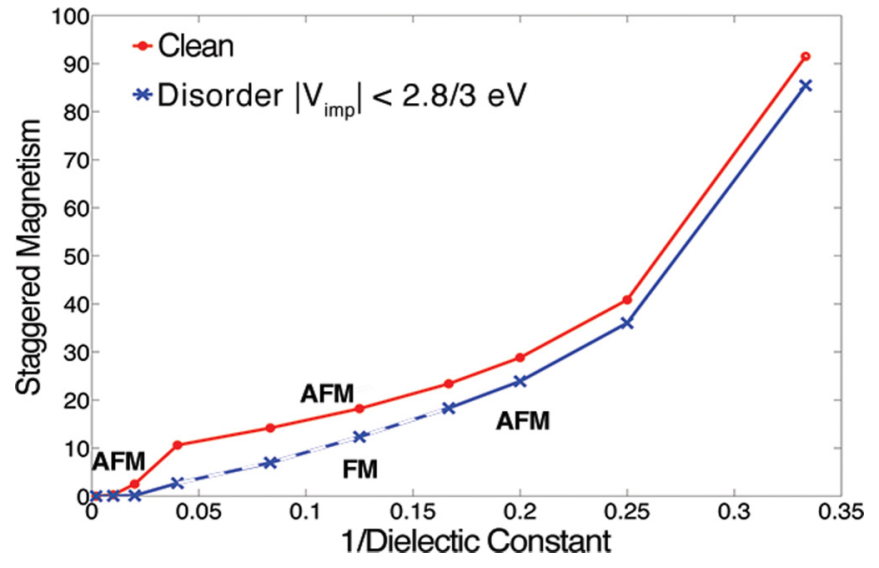

FIG. 6. Staggered magnetism as a function of dielectric constant $\kappa$. The clean system (upper line) shows AFM (solid line) coupled edges for all values within the $1 / \kappa=[0.33,0.002]$ range. However, a FM (dashed line) phase transition occurs between $1 / \kappa=$ $[0.167,0.04]$ after introducing the impurity landscape (lower line). For lower $\kappa$ values, electronic interaction effects become dominant over the impurities hence the system shows an AFM phase again.

\section{CONCLUSIONS}

To conclude, we have investigated the combined effects of electron-electron interactions and random potential fluctuations on the stability of edge states and magnetic phases. The electronic stability of edge states is found to be surprisingly robust against disorder due to electron-electron interactions. Moreover, as the disorder potential strength is increased, the system goes through an antiferromagnetic-to-ferromagnetic phase transition, in agreement with the experimental results of Ref. [22]. Although the possibility of such a transition is well known from previous calculations [37] for a charged system, here, the nanoribbon is charge neutral. Thus the magnetic transition is due to the formation of electron-hole puddles, i.e., local breaking of charge neutrality.

\section{ACKNOWLEDGMENTS}

This work was supported by The Scientific and Technological Research Council of Turkey (TUBITAK) under the 1001 Grant Project No. 114F331 and by Bilim Akademisi - The Science Academy, Turkey under the BAGEP program.
[1] A. H. C. Neto, F. Guinea, N. M. R. Peres, K. S. Novoselov, and A. K. Geim, Rev. Mod. Phys. 81, 109 (2009).

[2] K. S. Novoselov, A. K. Geim, S. V. Morozov, D. Jiang, Y. Zhang, S. V. Dubonos, I. V. Grigorieva, and A. A. Firsov, Science 306, 666 (2004).

[3] Y.-W. Son, M. L. Cohen, and S. G. Louie, Nature (London) 444, 347 (2006).

[4] M. Wimmer, I. Adagideli, S. Berber, D. Tomanek, and K. Richter, Phys. Rev. Lett. 100, 177207 (2008).

[5] J. Bundesmann, M. H. Liu, I. Adagideli, and K. Richter, Phys. Rev. B 88, 195406 (2013).
[6] A. D. Güçlü, P. Potasz, O. Voznyy, M. Korkusinski, and P. Hawrylak, Phys. Rev. Lett. 103, 246805 (2009).

[7] J. Fernandez-Rossier and J. J. Palacios, Phys. Rev. Lett. 99, 177204 (2007).

[8] A. D. Güçlü, arXiv:1510.05913v1 [cond-mat.mes-hall].

[9] E. H. Lieb, Phys. Rev. Lett. 62, 1927 (1989).

[10] M. Fujita, K. Wakabayashi, K. Nakada, and K. Kusakabe, J. Phys. Soc. Jpn. 65, 1920 (1996).

[11] K. Nakada, M. Fujita, G. Dresselhaus, and M. S. Dresselhaus, Phys. Rev. B 54, 17954 (1996). 
[12] O. V. Yazyev and M. I. Katsnelson, Phys. Rev. Lett. 100, 047209 (2008).

[13] K. Wakabayashi, M. Sigrist, and M. Fujita, J. Phys. Soc. Jpn. 67, 2089 (1998).

[14] B. Wunsch, T. Stauber, F. Sols, and F. Guinea, Phys. Rev. Lett. 101, 036803 (2008).

[15] A. Yamashiro, Y. Shimoi, K. Harigaya, and K. Wakabayashi, Phys. Rev. B 68, 193410 (2003).

[16] O. V. Yazyev, R. B. Capaz, and S. G. Louie, Phys. Rev. B 84, 115406 (2011).

[17] H. Feldner, Z. Y. Meng, A. Honecker, D. Cabra, S. Wessel, and F. F. Assaad, Phys. Rev. B 81, 115416 (2010).

[18] W. L. Wang, O. V. Yazyev, S. Meng, and E. Kaxiras, Phys. Rev. Lett. 102, 157201 (2009).

[19] J. Cao and S.-J. Xiong, Phys. Rev. B 88, 085409 (2013).

[20] W. Jaskolski, L. Chico, and A. Ayuela, Phys. Rev. B 91, 165427 (2015).

[21] A. R. Carvalho, J. H. Warnes, and C. H. Lewenkopf, Phys. Rev. B 89, 245444 (2014)

[22] G. Z. Magda, X. Jin, I. Hagymási, P. Vancsó, Z. Osváth, P. Nemes-Incze, C. Hwang, L. P. Biró, and L. Tapasztò, Nature 514, 608 (2014).

[23] X. Zhang, O. V. Yazyev, J. Feng, L. Xie, C. Tao,Y. C. Chen, L. Jiao, Z. Pedramrazi, A. Zettl, S. G. Louie, H. Dai, and M. F. Crommie, ACS Nano 7, 198 (2013).
[24] J. Kunstmann, C. Özdoğan, A. Quandt, and H. Fehske, Phys. Rev. B 83, 045414 (2011).

[25] P. Koskinen, S. Malola, and H. Häkkinen, Phys. Rev. Lett. 101, 115502 (2008)

[26] P. Koskinen, S. Malola, and H. Häkkinen, Phys. Rev. B 80, 073401 (2009)

[27] T. Wassmann, A. P. Seitsonen, A. M. Saitta, M. Lazzeri, and F. Mauri, Phys. Rev. Lett. 101, 096402 (2008).

[28] E. Rossi and S. Das Sarma, Phys. Rev. Lett. 101, 166803 (2008).

[29] S. Das Sarma, S. Adam, E. H. Hwang, and E. Rossi, Rev. Mod. Phys. 83, 407 (2011).

[30] G. Schubert and H. Fehske, Phys. Rev. Lett. 108, 066402 (2012).

[31] J. Martin, N. Akerman, G. Ulbricht, T. Lohmann, J. H. Smet, K. von Klitzing, and A. Yacoby, Nat. Phys. 4, 144 (2008).

[32] Y. Zhang, V. W. Brar, C. Girit, A. Zettl, and M. F. Crommie, Nat. Phys. 5, 722 (2009).

[33] M. Polini, A. Tomadin, R. Asgari, and A. H. MacDonald, Phys. Rev. B 78, 115426 (2008).

[34] M. Gibertini, A. Tomadin, F. Guinea, M. I. Katsnelson, and M. Polini, Phys. Rev. B 85, 201405(R) (2012).

[35] P. Potasz, A.D. Güçlü, and P. Hawrylak, Phys. Rev. B 82, 075425 (2010)

[36] A. D. Güçlü, M. Grabowski, and P. Hawrylak, Phys. Rev. B 87, 035435 (2013).

[37] J. Jung and A. H. MacDonald, Phys. Rev. B 79, 235433 (2009). 